\title{
FLEXURAL STRESS ANALYSIS OF RIGID PAVEMENTS USING AXI-SYMMETRIC AND PLANE STRAIN FEM
}

\author{
V.A. Sawant* \\ Applied Mechanics Department \\ Sardar Vallabhbhai National Institute of Technology, Surat India \\ M.S. Norazzlina \\ Civil Engineering Department, Universiti Malaysia Sarawak, Malaysia
}

Received 24 January 2007

\begin{abstract}
The design of pavement involves a study of soils and paving materials, their response under load for different climatic conditions. In the present study, an attempt has been made to compare stresses predicted using two finite element analyses. First analysis is based on the twodimensional plane strain assumption where as in second approach axi-symmetric condition is assumed to consider three-dimensional behavior of rigid pavement. The results are compared with flexural stresses obtained from conventional Portland Cement Association method. The computed flexural stresses obtained from axi-symmetric condition are found to be in close agreement with PCA method. Results of plane strain analysis show a fair agreement after application of an appropriate multiplication factor
\end{abstract}

Keywords: Pavement, axi-symmetric, plane strain

\section{INTRODUCTION}

Design of airport and highway pavement involves a study of soils and paving materials, their behavior under load, and climatic conditions. All pavements derive their ultimate support from the underlying sub-grade. Pavements are classified into flexible pavements and rigid pavements. Flexible pavements include primarily those pavements that have an asphalt concrete surface. Flexible pavement consists of four distinct layers, which includes a relatively thin wearing surface, a base course, sub base course, and compacted sub-grade. Rigid pavements are made up of Portland Cement Concrete and may or may not have a base course between the pavement and sub-grade.

The basic difference between two types of pavements, is the manner in which they distribute load over the surface. The rigid pavements because of its rigidity and high modulus of elasticity tend to distribute load over a wide area of soil. Thus a major portion of the structure capacity is imparted by slab itself. The major factor considered in the design of rigid pavements is the structural strength of concrete. Minor variations in sub-grade strength have little influence on

\footnotetext{
* Corresponding author e-mail: vasawant@yahoo.com
} 
the structural capacity of pavement. In the case of flexible pavements, the strength is the result of building thick layers and thereby distributing the load over the sub-grade, rather than by bending action of the slab. The thickness of the pavement is influenced by the strength of the sub-grade.

Most rigid pavement design techniques are based on theoretical stresses in elastic slabs, modified by field experience and appropriate safety factors. According to theoretical analysis the magnitude of stresses in pavements depends also upon the modulus of sub-grade reaction, the modulus of elasticity of concrete and Poisson's ratio of the concrete. The development of equilibrium theories, stress and displacement can be evaluated for a pavement system. Westergard and Hogg introduced the mathematical solution of bending moment and bending stress for a pavement layer supported by an idealized sub-grade. Conventional design approaches for Rigid Pavement (and even flexible pavement) are based on the assumption that Pavement is resting on a homogeneous mass, which is not true. Stresses calculated with this assumption may be erratic when base material is not homogeneous. A more rational approach would be to adopt finite element analysis, where non- homogeneous nature of base materials can be properly accounted. Finite element analysis is a powerful tool in analyzing for almost any combination of load environment till an optimum design is reached.

In the present study, an attempt has been made to compare stresses predicted using two finite element analyses. First analysis is based on the two-dimensional plane strain assumption where as in the second approach axi-symmetric condition is assumed for considering threedimensional behavior of rigid pavement. The pavement and sub-grade are modeled using 8 noded isoparametric elements. Flexural stresses obtained from two finite element analysis are compared with the charts of conventional Portland Cement Association method of design. The study shows a fair agreement between the results of finite element analyses and conventional method of design.

\section{FINITE ELEMENT ANALYSIS}

The pavement and sub-grade are modeled using 8 noded rectangular elements having Young's modulus E, and Poisson's ratio $v$. The coordinates $(x, z)$ of a point within the elements are expressed in terms of local coordinates $(\xi, \eta)$ of the elements as

$$
x=\sum_{i=1}^{8} N_{i} x_{i} \quad \text { and } \quad z=\sum_{i=1}^{8} N_{i} z_{i}
$$

where, $\left(\mathrm{x}_{\mathrm{i}}, \mathrm{z}_{\mathrm{i}}\right)$ are nodal coordinates of the element and $\mathrm{N}_{\mathrm{i}}$, defines shape functions. Same shape functions are used to define horizontal displacement $\mathrm{u}$ and vertical displacement $\mathrm{w}$, within the element.

$$
u=\sum_{i=1}^{8} N_{i} u_{i} \quad \text { and } \quad w=\sum_{i=1}^{8} N_{i} w_{i}
$$

For the node numbering shown in Fig. 1, shape functions are,

$$
\begin{aligned}
& N_{i}=\frac{1}{4}\left(1+\xi_{i} \xi\right)\left(1+\eta_{i} \eta\right)\left(\xi_{i} \xi+\eta_{i} \eta-1\right) \\
& \text { for } \quad i=1,3,5,7 \\
& N_{i}=\frac{1}{2}\left(1-\xi^{2}\right)\left(1+\eta_{i} \eta\right) \text { for } i=2 \text { and } 6
\end{aligned}
$$




$$
N_{i}=\frac{1}{2}\left(1+\xi_{i} \xi\right)\left(1-\eta^{2}\right) \quad \text { for } i=4 \text { and } 8
$$

where, $\left(\xi_{i}, \eta_{i}\right)$ are local coordinates of $\mathrm{i}^{\text {th }}$ node.

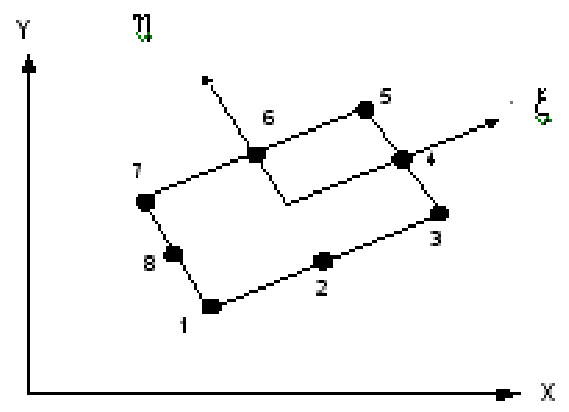

Fig. 1: Eight noded element.

The nodal displacement vector, $\{\delta\}$, given by,

$$
\{\delta\}^{T}=\left\{u_{1}, w_{1}, u_{2}, w_{2}, \ldots \ldots, u_{8}, w_{8}\right\}
$$

\section{PLANE STRAIN ANALYSIS}

The strain components $\varepsilon_{\mathrm{x}}, \varepsilon_{\mathrm{z}}$ and $\gamma_{\mathrm{xz}}$ are related to displacements $\mathrm{u}$ and $\mathrm{v}$ as follows,

$$
\{\varepsilon\}=\left\{\begin{array}{c}
\varepsilon_{x} \\
\varepsilon_{z} \\
\gamma_{x z}
\end{array}\right\}=\left\{\begin{array}{c}
\frac{\partial u}{\partial x} \\
\frac{\partial w}{\partial z} \\
\frac{\partial u}{\partial z}+\frac{\partial w}{\partial x}
\end{array}\right\}=[B]\{\delta\}_{e}
$$

where, $[\mathrm{B}]$, is strain-displacement transformation matrix, $\{\varepsilon\}$, is strain vector and, $\{\delta\}$, is vector of unknown displacements.

Stress strain relationship is given as,

$$
\{\sigma\}=\left\{\begin{array}{c}
\sigma_{x} \\
\sigma_{z} \\
\tau_{x z}
\end{array}\right\}=[D]\left\{\begin{array}{c}
\varepsilon_{x} \\
\varepsilon_{z} \\
\gamma_{x z}
\end{array}\right\}=[D]\{\varepsilon\}
$$

in which, $\{\sigma\}$, is stress vector, and $v$ is the Poisson's ratio, and [D], is constitutive relation matrix given as

$$
[D]=\frac{E}{(1-2 v)(1+v)}\left[\begin{array}{ccc}
1-v & v & 0 \\
v & 1-v & 0 \\
0 & 0 & \frac{1-2 \nu}{2}
\end{array}\right]
$$

Element stiffness matrix, $[\mathrm{K}]_{\mathrm{e}}$, is given by, 


$$
[k]_{e}=\int_{-1}^{1} \int_{-1}^{1}[B]^{T}[D][B]|J| d \xi d \eta
$$

Load vector, $\{\mathrm{F}\}$, for uniformly distributed load, $\mathrm{q}_{\mathrm{z}}$, acting on the element can be obtained as,

$$
\{F\}=\int_{-1}^{1}[N]^{T}\left\{\begin{array}{c}
0 \\
q_{z}
\end{array}\right\} a d \xi
$$

Integration is carried out numerically with respect to $\xi$ and $\eta$, using Gauss quadrature.

\section{AXI-SYMMETRIC ANALYSIS}

The strain components $\varepsilon_{\mathrm{r}}, \varepsilon_{\theta} \varepsilon_{\mathrm{z}}$ and $\gamma_{\mathrm{r} \theta}$ are related to displacements $\mathrm{u}$ and $\mathrm{v}$ as follows,

$$
\{\varepsilon\}=\left\{\begin{array}{c}
\varepsilon_{r} \\
\varepsilon_{\theta} \\
\varepsilon_{z} \\
\gamma_{r z}
\end{array}\right\}=\left\{\begin{array}{c}
\frac{\partial u}{\partial r} \\
\frac{u}{r} \\
\frac{\partial w}{\partial z} \\
\frac{\partial u}{\partial z}+\frac{\partial w}{\partial r}
\end{array}\right\}=[B]\{\delta\}_{e}
$$

where, $[\mathrm{B}]$, is strain-displacement transformation matrix, $\{\varepsilon\}$, is strain vector and, $\{\delta\}$, is vector of unknown displacements.

Stress strain relationship is given as,

$$
\{\sigma\}=\left\{\begin{array}{c}
\sigma_{r} \\
\sigma_{\theta} \\
\sigma_{z} \\
\tau_{r z}
\end{array}\right\}=[D]\left\{\begin{array}{c}
\varepsilon_{r} \\
\varepsilon_{\theta} \\
\varepsilon_{z} \\
\gamma_{r z}
\end{array}\right\}=[D]\{\varepsilon\}
$$

in which, $\{\sigma\}$, is stress vector, and $v$ is the Poisson's ratio, and [D], is constitutive relation matrix given as

$$
[D]=\frac{E}{(1-2 v)(1+v)}\left[\begin{array}{cccc}
1-v & v & v & 0 \\
v & 1-v & v & 0 \\
v & v & 1-v & 0 \\
0 & 0 & 0 & \frac{1-2 v}{2}
\end{array}\right]
$$

Element stiffness matrix, $[\mathrm{K}]_{\mathrm{e}}$, is given by,

$$
[k]_{e}=2 \pi \int_{-1}^{1} \int_{-1}^{1}[B]^{T}[D][B]|J| r \quad d \xi d \eta
$$

Load vector, $\{\mathrm{F}\}$, for uniformly distributed load, $\mathrm{q}_{\mathrm{z}}$, acting on the element can be obtained as, 


$$
\{F\}=\int_{-1}^{1}[N]^{T}\left\{\begin{array}{c}
0 \\
q_{z}
\end{array}\right\} 2 \pi \operatorname{ard} d \xi
$$

Integration is carried out numerically with respect to $\xi$ and $\eta$, using Gauss quadrature.

\section{GEOMETRY AND MATERIAL PROPERTIES}

The assumed dimensions of the slab and subgrade are shown in Figure 2. Due to symmetric loading applied on pavement surface, only one half of the pavement is taken for analysis. The thickness of slab will be varied between 6" to 22". The modulus of elasticity of concrete and Poisson's ratio of the concrete are input parameters. Typical values of those are $4000000 \mathrm{psi}$ and 0.15 . Normally for the pavement analysis, sub-grade reaction will be used. But present analysis needs the modulus of elasticity, $\mathrm{E}_{\mathrm{s}}$, and Poisson's ratio of the soil. Poisson's ratio of the soil is assumed as 0.25. Following relationship given by Bowles (1988), is used to estimate $\mathrm{E}_{\mathrm{s}}$ from $\mathrm{K}_{\mathrm{s}}$.

$$
E_{s}=K_{s} B\left(1-\mu^{2}\right)
$$

Five values of subgrade reaction modulus are considered in the present study. Table 1 presents subgrade reaction modulus and equivalent modulus of elasticity.

Table 1: Subgrade reaction and modulus of elasticity.

\begin{tabular}{ll}
\hline $\mathbf{k}_{\mathbf{s}}(\mathbf{p c i})$ & $\mathbf{E}_{\mathbf{s}}(\mathbf{p s i})$ \\
\hline 50 & 6750 \\
100 & 13500 \\
200 & 27000 \\
300 & 40500 \\
\hline
\end{tabular}

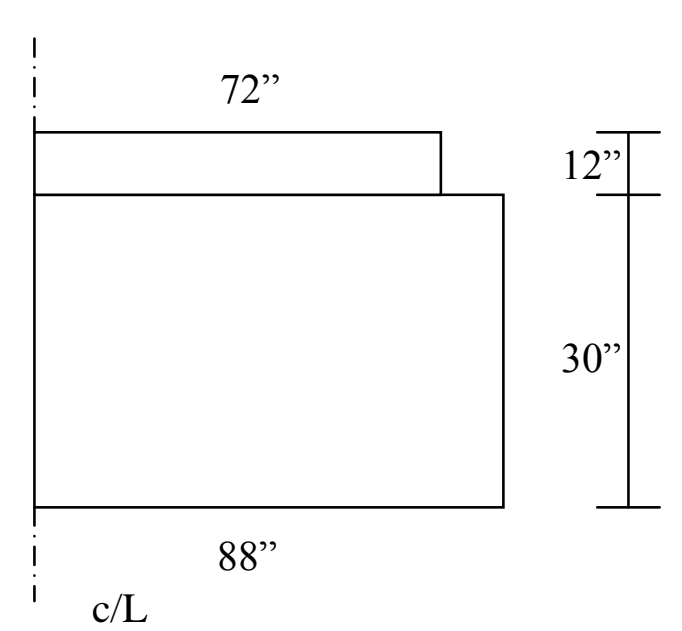

Fig. 2: Half cross section of pavement slab.

Total load on tire is $44000 \mathrm{lb}$, and tire pressure on dual wheels 24 " c-c is taken as $136.6 \mathrm{psi}$. If the effect of the tire wall is ignored, the contact pressure between the tire and pavement must be equal to tire pressure. In many cases it is assumed that contact pressures are uniform over the 
circular imprint area. For total load, $\mathrm{P}$, on the tire, and tire pressure, p, radius of contact, a, is given as follows.

$$
a=\sqrt[4]{\frac{P}{p \pi}}=10.12572
$$

The following boundary conditions are assumed for both type of analysis. Horizontal displacements are zero along center line and outer edge. Vertical displacement is zero along bottom line. Stiffness matrix for each element is assembled into global stiffness matrix. Assembled global stiffness matrix and Force vector will be modified for known boundary conditions and then are to be solved for unknown displacement by Gauss Elimination. From displacements stresses in each element will be worked out. A parametric study is carried out to study the effect of pavement thickness, and subgrade reaction modulus.

\section{RESULTS}

Table 2 compares the results of the two analyses for subgrade reaction 50 pci with slabthickness varied between 10" to 18". Maximum flexural stresses obtained from PCA method are also presented for comparison. It is clear from Table 2 that results obtained by plane strain finite element analysis are on higher side. This can be attributed to two-dimensional plane strain assumption, where it is assumed that length of the tire-pressure in perpendicular direction is infinity.

Table 2: Comparison of maximum flexural stress.

\begin{tabular}{llll}
\hline Thickness & FEM & Axi-FEM & PCA \\
\hline 10 & 1515.869 & 297.6950 & 377.55 \\
12 & 1277.531 & 221.1313 & 340.00 \\
14 & 1053.649 & 169.9195 & 260.00 \\
16 & 866.5168 & 134.5611 & 185.71 \\
18 & 718.6769 & 109.4822 & 151.02 \\
\hline
\end{tabular}

To have a real comparison between three-dimensional and two-dimensional nature a multiplying factor is computed based on the theory of beams on elastic foundation. Factor is the ratio of response to uniformly distributed load of finite length (2a) to that of infinite length.

$$
M . F .=1-e^{-\beta a} \cos (\beta a)
$$

where $\beta=\sqrt[4]{\frac{k}{4 E I}}$

A factor of safety 2 is also considered for plane strain analysis. For axi-symmetric condition a smaller factor of safety 1.5 is used. Flexural stresses after applying multiplying factor and factor of safety are reported in Table 3. Same result is also presented in graphical form in Figure 3 for comparison between FEM and PCA method.

The variation in flexural stress with slab-thickness for different values of subgrade reaction modulus is presented in Figure 4 for plane strain condition. Result shows the expected trend with discrepancies at some points. For example for k equal to 50 and 100, point of discrepancy 
is observed at 22", whereas for k equal to 200 and 300 , same is at 20 ". For k equal to 500, it is shifted to 18". This shows that point of discrepancy is shifting towards lower value with increase in subgarde reaction modulus. This appears to be a phenomenon like resonance in dynamic problem, hence needs more investigation. Variation in flexural stress for axi-symmetric condition as described in Figure 5 also indicate similar trend.

Table 3: Comparison of flexural stresses after conversion.

\begin{tabular}{cccc}
\hline Thickness & FEM & Axi-FEM & PCA \\
\hline 10 & 424.5606 & 446.5425 & 377.55 \\
12 & 312.5281 & 331.6970 & 340.00 \\
14 & 229.8362 & 254.8793 & 260.00 \\
16 & 171.1187 & 201.8417 & 185.71 \\
18 & 129.9884 & 164.2233 & 151.02 \\
\hline
\end{tabular}

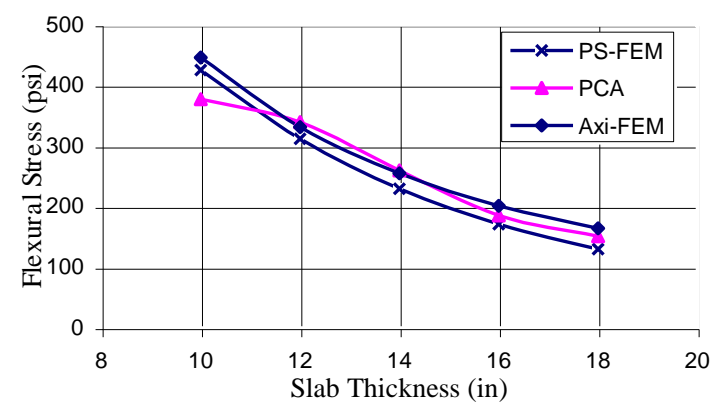

Fig. 3: Comparison between FEM and PCA.

The deflected shape of pavement top layer is plotted in Figure 6 for plane strain condition and in Figure 7 for axi-symmetric condition. Displacements obtained from plane strain analysis are higher as compared to those of axi-symmetric analysis. For both conditions, deflections are reduced with increase in slab-thickness.

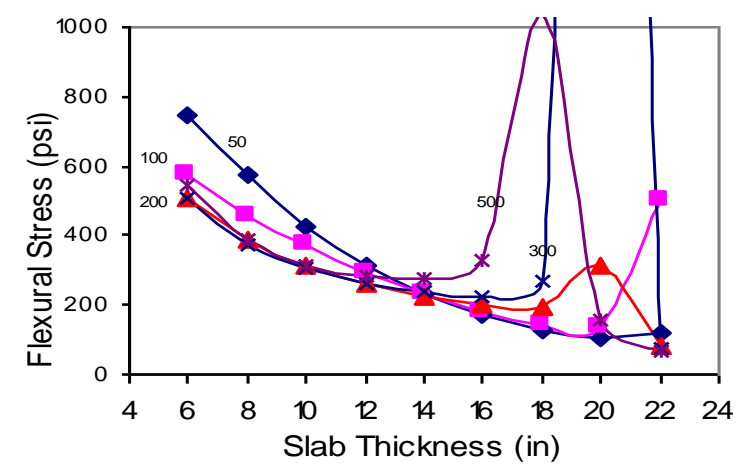

Fig. 4: Flexural stress in plane strain condition. 


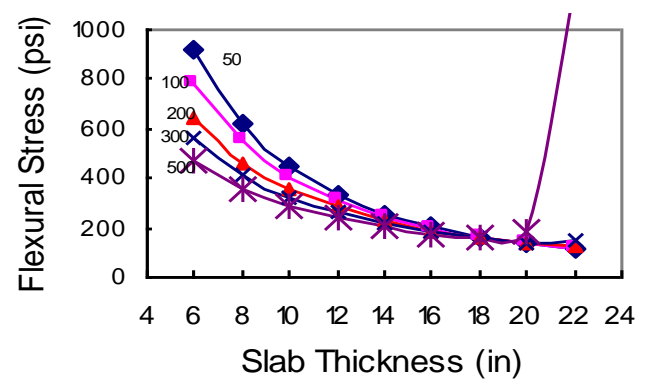

Fig. 5: Flexural stress in axi-symmetric condition.

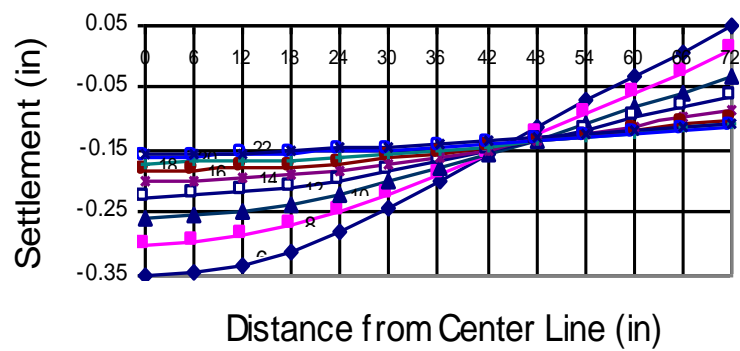

Fig. 6: Deflection of pavement plane strain FEM.

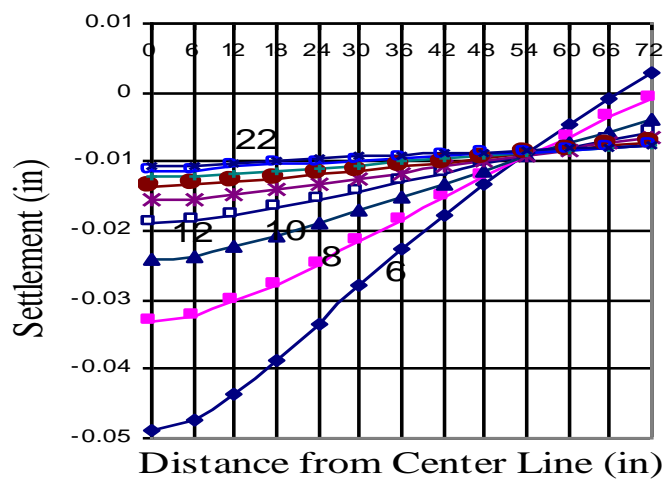

Fig. 7: Deflection of pavement axi-symmetric FEM.

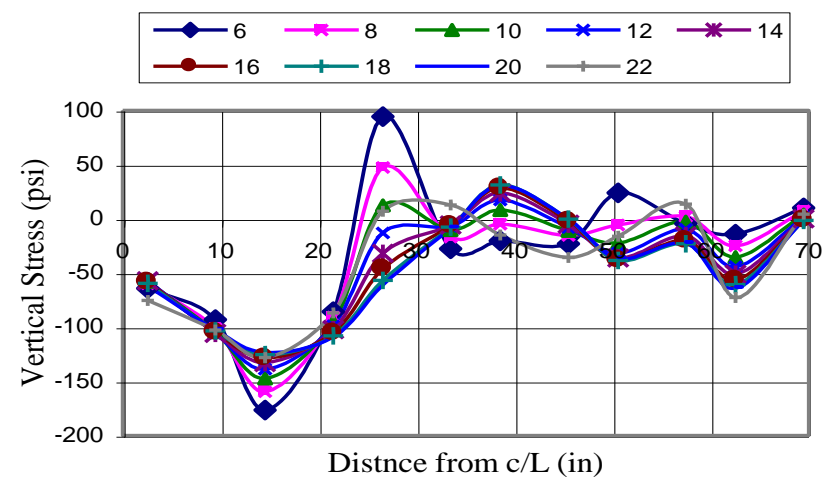

Fig. 8: Vertical stress in pavement plane strain FEM. 

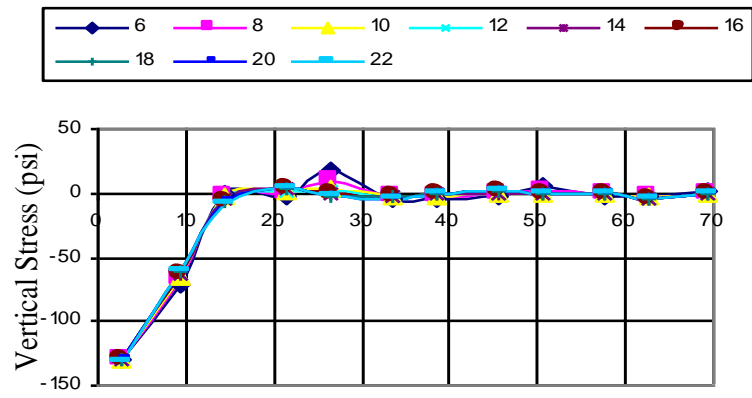

Distnce from c/L (in)

Fig. 9: Vertical stress in pavement axi-symmetric FEM.

Figure 8 describes vertical stress distribution in the concrete pavement for plane strain condition with various slab thickness, for the case of subgrade modulus 50 pci. Plot indicates vertical stresses are higher in thinner slab. Maximum vertical stress decreases with increase in slab thickness. Vertical stress distribution for axi-symmetric condition is shown in Figure 9. As compare to plane strain condition, less variation is observed with slab thickness.

\section{CONCLUSION}

Following conclusion are drawn from present study.

1. Results of finite element analysis for axi-symmetric condition show good agreement with conventional PCA method of design.

2. Results of plane strain analysis are on higher side as compare to axi-symmetric condition. The study shows a fair agreement between the results of plane strain finite element analysis and conventional method of design only after application of an appropriate multiplication factor.

3. The variation in flexural stress with slab-thickness shows the expected trend with discrepancies at some points. Maximum flexural stress decreases with increase in slab thickness and subgrade reaction modulus.

4. As slab-thickness increases, deflections and maximum vertical stresses are reduced.

\section{ACKNOWLEDGEMENT}

The authors acknowledge the financial support rendered by Unimas through the Short Term Research Grant No. 02(47)/455/2004(192).

\section{REFERENCES}

1. Bowles Joseph E. (1988), Foundation Analysis and Design, 4th edition, McGraw Hill.

2. Putuappa, C.G., Muthu, K.U., Anjaneya Murthy, D.S., and Veeraragavan, A. (2002), Flexural Stress Analysis of Rigid Pavements Using NISA, Proceedings of National Seminar on Road Transportation in India: Emerging Trends and Techniques, September 12-13, IIT Kharagpur, India: 1.13-1.20

3. Yoder, E.J. and Witzak, M.W. (1975), Principles of Pavement Design, 2nd edition, John Wiley and Sons. 